\title{
Primary bone mantle cell lymphomas with multiple vertebral compression fractures: A case report
}

\author{
PENG YANG ${ }^{*}$, JUN LIN $^{*}$, HAO LIU, HAO SHEN and HUI-LIN YANG \\ Department of Orthopaedic Surgery, The First Affiliated Hospital of Soochow University, Suzhou, Jiangsu 215006, P.R. China
}

Received January 14, 2015; Accepted February 11, 2016

DOI: $10.3892 / \mathrm{ol} .2017 .5553$

\begin{abstract}
Mantle cell lymphoma (MCL) is a mature B-cell non-Hodgkin lymphoma. Patients with MCL generally present with extensive lymphadenopathy and extranodal involvement. To the best of our knowledge, no cases of primary bone MCL with multiple vertebral compression fractures have been reported in the literature thus far. The current study reports the case of a 75-year-old Chinese male patient presenting with lower back pain for 1 year. B symptoms were not observed in the patient's medical history. X-ray revealed osteoporosis, and mixed osteolytic and osteosclerotic lesions in the T10 and L1 vertebrae with decreased height. Computed tomography of the spine showed multiple vertebral compression fractures, particularly at T10 and L1. Furthermore, magnetic resonance imaging demonstrated a diffuse homogeneous abnormal signal with multiple vertebral compression fractures. The patient underwent vertebral biopsy and percutaneous balloon kyphoplasty. Histopathological examination of the biopsy revealed abnormal, diffuse, atypical, small-sized, slightly irregular lymphocytes with condensed chromatin. Immunohistochemical staining showed $\mathrm{CD}^{+}, \mathrm{CD} 20^{+}$and cyclin $\mathrm{D}^{+}$. Thus, the patient was diagnosed with primary bone MCLs and chemotherapy was administered. During the 2-month follow-up, the patient remained in a good clinical condition. The present study reports a rare case of primary osseous MCL and a review of the literature.
\end{abstract}

\section{Introduction}

Mantle cell lymphoma (MCL) is a mature B-cell non-Hodgkin lymphoma (NHL) (1). MCL is characterized by atypical small lymphoid cells with wide mantles around benign germinal

Correspondence to: Professor Hui-Lin Yang, Department of Orthopaedic Surgery, The First Affiliated Hospital of Soochow University, 188 Shizi Street, Suzhou, Jiangsu 215006, P.R. China

E-mail: hlyangsuzhou@gmail.com

${ }^{*}$ Contributed equally

Key words: mantle cell lymphoma, tumor, vertebrae, compression fractures, treatment centers, therefore MCL is known as a mantle zone lymphoma (2). MCL accounts for 3-10\% of adult NHLs (3). The median age of patients presenting with MCL is in the seventh decade of life, and there is a striking male predominance (2:1). MCL is associated with the chromosomal translocation $\mathrm{t}(11,14)(\mathrm{q} 13 ; \mathrm{q} 32)$, which leads to cyclin D1 overexpression (3). Patients may present with pancytopenia or leukemia with extensive leukocytosis (4). Patients generally have Ann Arbor stage III/IV disease at the time of diagnosis, and commonly present with extensive lymphadenopathy and extranodal involvement, particularly bone marrow, gastrointestinal tract, liver, spleen or Waldeyer's ring involvement (5). MCL is particularly involved in the gastrointestinal system and may mimic other diseases (6), which may cause a delay in diagnosis and treatment. In past years, MCL had a poor prognosis, with a 5-year overall survival rate of $27 \%$ (7). Due to the introduction of novel drugs and therapeutic options, the effect of treatment for MCL has shown significant improvements, with a current 5-year survival rate of 50-75\% (8). To the best of our knowledge, no cases of primary bone MCL, involving either single or multiple bones, have been reported in the literature thus far. The clinical features of the disease may mimic other diseases, which can cause a delay in the diagnosis and treatment. The current study presents a rare case of primary MCL of the spine in a 75-year-old Chinese male patient.

\section{Case report}

A 75-year-old Chinese male patient presented to the The First Affiliated Hospital of Soochow University (Suzhou, China) on September, 182014 with lower back pain for 1 year, without a history of traumatic events. The pain was described as moderately pricking but not radiating, and it was exacerbated by movement and coughing. B symptoms were not observed in the patient's medical history. Furthermore, there was no history of bleeding manifestations, or respiratory, cardiovascular, urinary or gastrointestinal symptoms. The patient had undergone an appendectomy 40 years prior. He did not suffer from diabetes, and did not smoke or consume excess alcohol. The findings of the physical examination were not significant: No lymphadenopathy, petechiae or ecchymoses were identified; the patient's spleen and liver were not palpable; and pain was only felt upon spinal percussion of the T10 and T12 vertebrae. In addition, the examination revealed no hypoesthesia, weakness or spasticity. Blood test results were as follows: Hemoglobin, $10.5 \mathrm{~g} / \mathrm{dl}$ (normal range, 13.0-17.5 g/dl); white blood cells, 
A
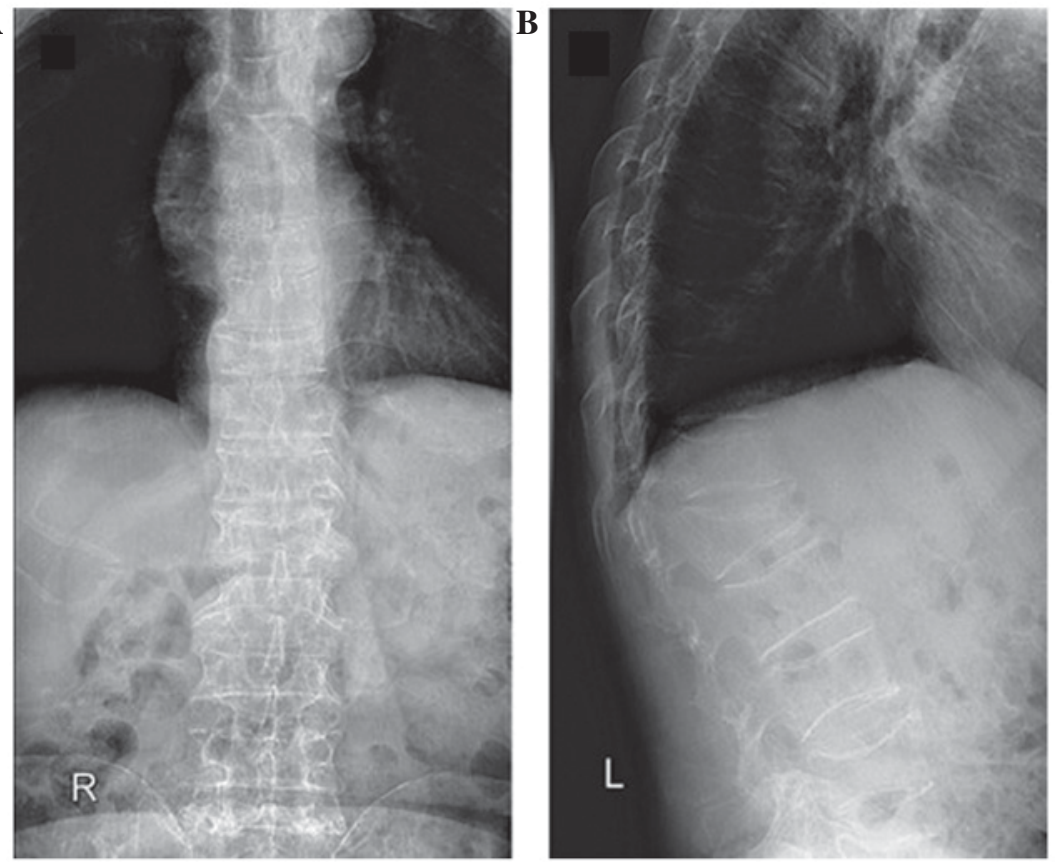

Figure 1. (A) Anterior/posterior and (B) oblique spinal X-ray showing severe osteoporosis, and mixed osteolytic and osteosclerotic lesions, particularly in T10 and L1, with reduced height prior to surgery. No periosteal reaction was observed.
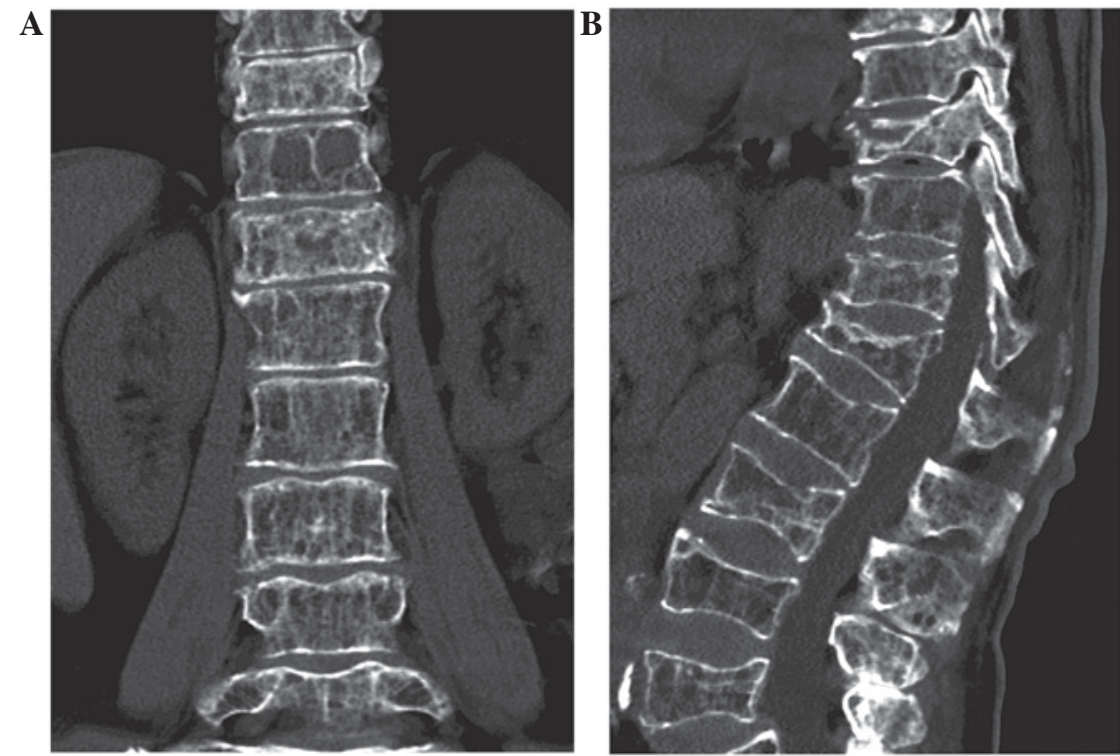

Figure 2. Spinal computed tomography (CT) scan indicating multiple vertebral compression fractures, particularly in T10 and L1. (A) Coronal CT images showing severe osteoporosis and osteolytic, expansile lesions in T11. (B) Sagittal CT images showing multiple severe vertebrae fractures and cortical bone thinning.

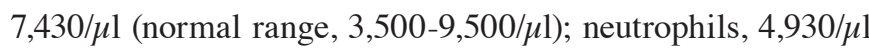
(normal range, 1,800-6,300/ $\mu$ ); lymphocytes, $1,860 / \mu 1$ (normal range, 1,100-3,200/ $/$ l); and platelets, 246,000/ $/ \mu \mathrm{l}$ (normal range, $125,000-350,000 / \mu \mathrm{l})$. Slightly increased C-reactive protein levels (13 mg/l; normal range, $0-8 \mathrm{mg} / \mathrm{l}$ ), erythrocyte sedimentation rate $(38 \mathrm{~mm} / \mathrm{h}$; normal range, $0-15 \mathrm{~mm} / \mathrm{h})$ and ferritin levels (412.1 ng/ml; normal range, 22.0-322.0 ng/ml) were observed. Lactic dehydrogenase (LDH) was $133 \mathrm{U} / 1$ (normal range, 100-225 U/l). However, the bone mineral density test T-score was -3.5 (normal range, -1.0-1.0), indicating severe osteoporosis. The Bence-Jones protein urine test was negative. A computed tomography (CT) scan of the neck, chest and abdomen showed no abnormalities. Spinal X-ray (Ysio Digital X-ray system; Siemens AG, Munich, Germany) revealed osteoporosis (Fig. 1A and B), and mixed osteolytic and osteosclerotic lesions in T10 and L1 with decreased height. A spinal CT scan (SOMATOM Sensation 64; Siemens AG) identified multiple vertebral compression fractures, particularly in T10 and L1 (Fig. 2A and B). Sagittal magnetic resonance imaging (MRI; Signa HDxt 3.0T; GE Healthcare Life Sciences, Little Chalfont, UK) demonstrated a diffuse homogeneous abnormal signal with multiple vertebral compression fractures (Fig. 3A-C). Similarly, T1-weighted MRI showed multiple vertebral compression fractures with a low signal intensity. T10 and L1 appeared to harbor the most 

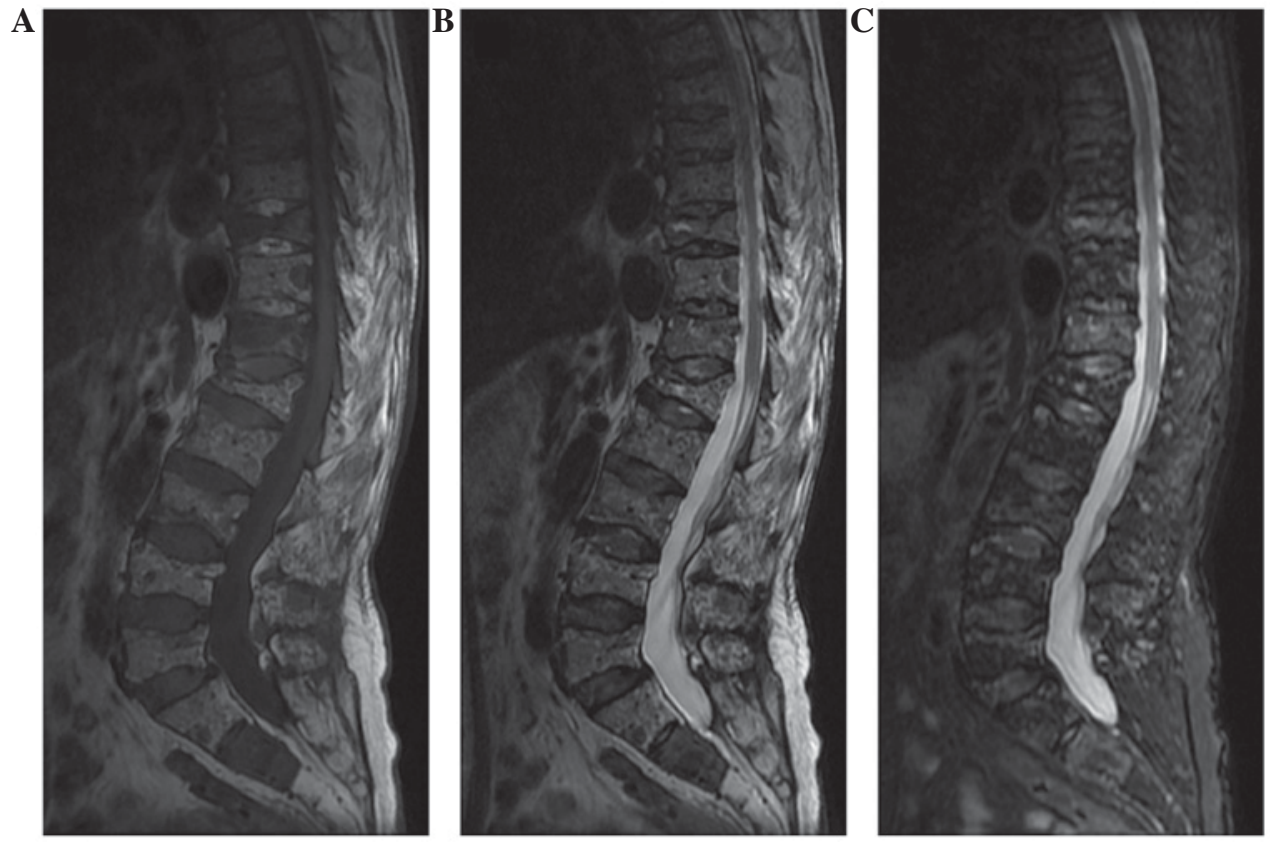

Figure 3. Sagittal magnetic resonance imaging (MRI) showing a diffuse homogeneous abnormal signal with multiple vertebral compression fractures. (A) T1-weighted MRI revealing multiple vertebral compression fractures with a low signal intensity, the most severe of which were observed in T10 and L1. (B) T2-weighted MRI also indicating multiple vertebral fractures with a mildly high signal intensity. (C) On the short tau inversion recovery sequences, MRI revealed a diffuse homogeneous abnormal signal. A mildly high signal intensity was observed in T10 and T12.
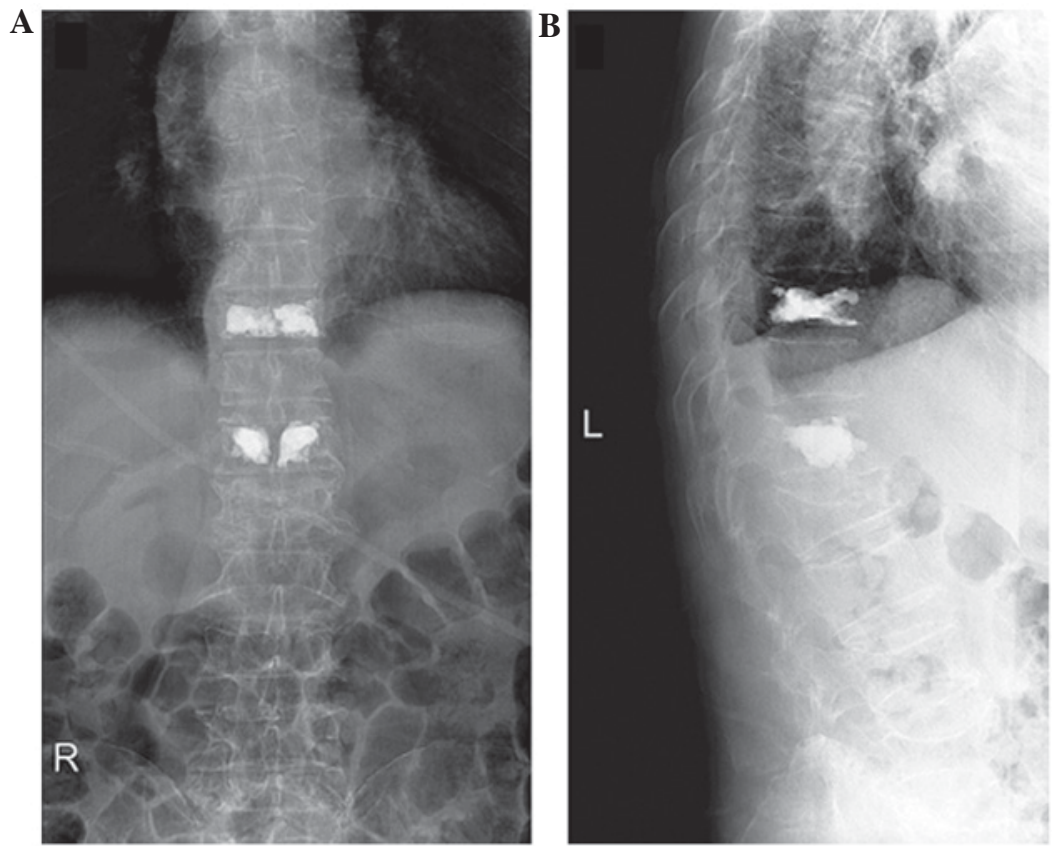

Figure 4. Postoperative (A) anteriorposterior and (B) oblique radiographs, revealing satisfying fracture reduction and cement distribution.

severe fractures. T2-weighted MRI also indicated multiple vertebral fractures with a mildly high signal intensity. On the short tau inversion recovery sequences, MRI revealed a diffuse homogeneous abnormal signal. A mildly high signal intensity was detected in T10 and T12, suggesting that T10 and T12 were responsible for the pain. The initial clinical diagnosis was pathological vertebral compression fractures and osteoporosis. Differential diagnosis typically includes osteoporotic vertebral compression fractures, multiple myeloma, osteomyelitis, lymphoma, metastasis and primary bone sarcoma. To confirm the proposed diagnosis, the patient underwent vertebral biopsy on September, 22 2014, and percutaneous balloon kyphoplasty (PKP) of T10 and T12. Postoperative radiographs (Fig. 4A and $\mathrm{B})$ revealed satisfying fracture reduction and cement distribution. Tumor samples were fixed in formalin (Sangon Biotech Co. Ltd., Shanghai, China). Subsequently, the samples were decalcified with $20 \%$ ethylenediamine tetra-acetic acid (Sangon Biotech Co. Ltd), dehydrated using a graded ethanol (Sangon Biotech Co. Ltd) series and paraffin (Sangon Biotech Co. Ltd.) embedded. Tumor tissue sections (4- $\mu$ m thick) were 

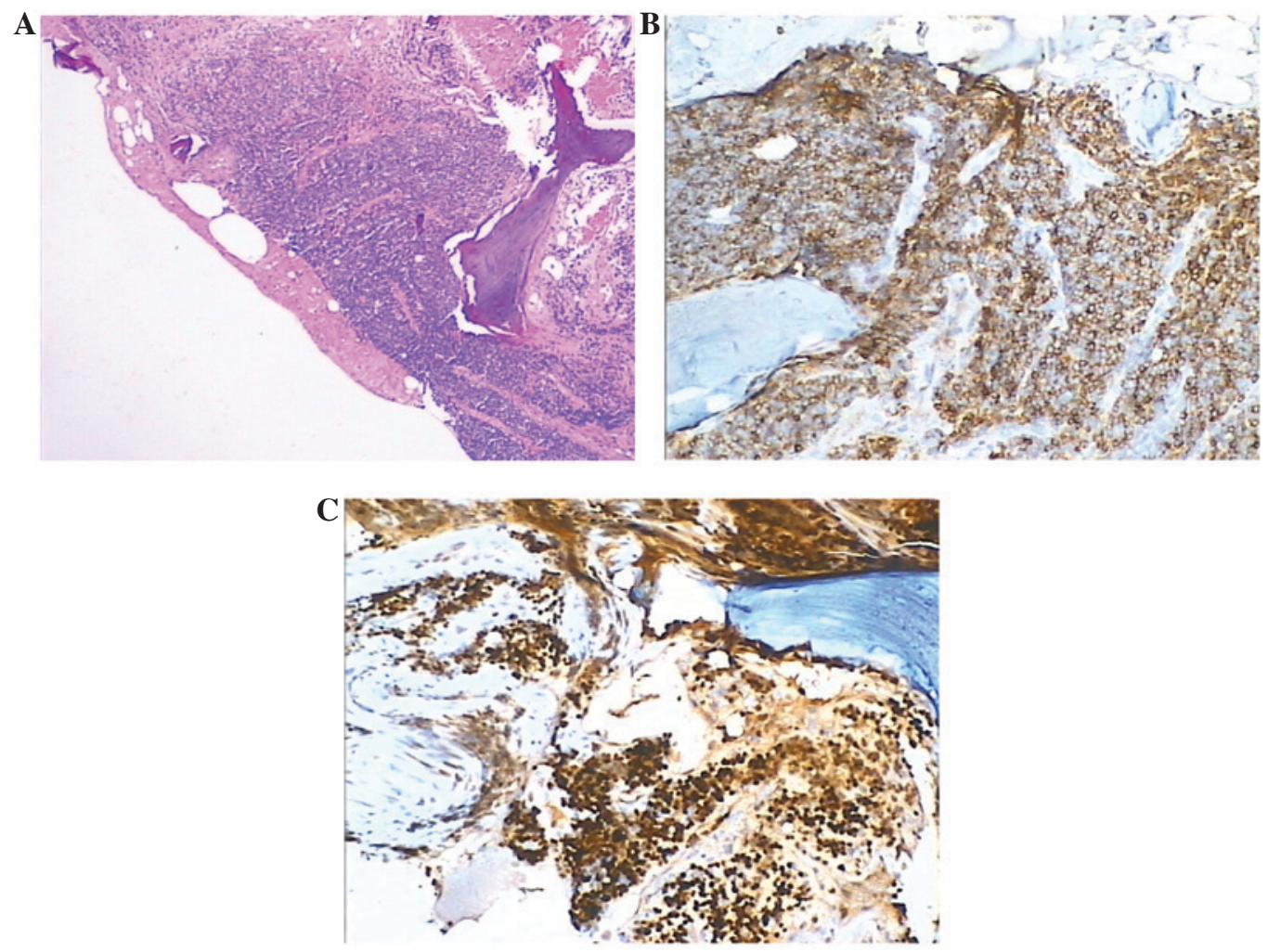

Figure 5. Histological and immunohistochemical staining revealing typical characteristics of mantle cell lymphoma. (A) Histological staining showing abnormal, diffuse, atypical, small-sized, slightly irregular lymphocytes with condensed chromatin (hematoxylin and eosin staining; magnification, x100). Immunohistochemical staining showing tumor cells with (B) $\mathrm{CD} 20^{+}$and (C) cyclin $\mathrm{D}^{+}$(diaminobenzidine and hematoxylin staining; magnification, $\mathrm{x} 400$ ).

sliced, deparaffinized in xylene (Sangon Biotech Co. Ltd.) and stained with hematoxylin and eosin (Sangon Biotech Co. Ltd.). The tissue sections were visualized by microscopy (Eclipse E600; Nikon Corporation, Tokyo, Japan). Histopathological examination of the vertebral biopsy showed abnormal, diffuse, atypical, small-sized, slightly irregular lymphocytes with condensed chromatin (Fig. 5A). Immunohistochemical staining was performed using the EnVision two-step staining method (Dako, Glostrup, Denmark). Deparaffinization, rehydration and epitope retrieval were performed using PT Link kit (Dako). The primary antibodies used were monoclonal mouse anti-cluster of differentiation (CD) 20 (catalog no., F0799; Dako) and monoclonal rabbit anti-cyclin D1 (catalog no., IR083; Dako), at a dilution of 1:100. Diaminobenzidine and hematoxylin staining were achieved using the autostainer system (Autostainer Link 48; Dako). Immunohistochemistry demonstrated that the tumor cells expressed CD20 and cyclin D1 (Fig. 5B and $\mathrm{C}$ ). The histopathological and immunohistochemical features of the vertebral bone were concordant with MCL. Following PKP surgery, the lower back pain was immediately relieved. The patient was then referred to the Department of Hematology with the diagnosis of MCL stage IV, according to the Ann Arbor classification (9), and was dicharged on September, 25 2014. An R-CHOP chemotherapy regime was administered to the patient 4 weeks following surgery. In total, the patient received 8 cycles of rituximab (700 $\mathrm{mg}$ on day 1), cyclophosphamide (1.4 $\mathrm{g}$ on day 2 ), doxorubicin (140 $\mathrm{mg}$ on day 2 ), vincristine (4 mg on day 2 ) and dexamethasone (15 mg on day 2). During 1 year follow-up, the patient remained in good clinical condition, only complaining of slight back pain.
Written informed consent was obtained from the patient for the publication of the present study.

\section{Discussion}

In 1964, Lennert first described MCL. Lennert termed the disease 'centrocytic lymphoma', as he considered these tumors to be the neoplastic counterpart of centrocytes located in germinal centers of lymphoid follicles (10). Various other terms were used to describe the condition in the following decades, until, in 1992, Banks et al (11) proposed the term MCL for this neoplasm. MCL subsequently became widely accepted, and has been adopted in the Revised European-American Classification of Lymphoid Neoplasms (12) and the World Health Organization classification of lymphoid neoplasms (13).

MCL is typically diagnosed in patients at an advanced stage of the disease (Ann Arbor stage III/IV), and the common symptoms include generalized lymphadenopathy, and involvement of the spleen, liver and bone marrow. The majority of MCLs develop within lymph nodes; the extranodal form is considerably rare. The most frequently affected extra-lymphatic sites are the gastrointestinal tract, Waldeyer's ring and nasopharynx (14). In the current case, the patient presented with pain in the lower back. No lymphadenopathy or hepatosplenomegaly were identified. Histopathological and immunohistochemical examination of the biopsy revealed primary MCL of the spine.

Primary bone lymphoma is rare and primary bone MCL is even more rare. To the best of our knowledge, no cases of 
MCL, involving either single or multiple bones, have been reported in the literature thus far. The patient in the present study exhibited multiple lumbar vertebral bone involvement. Thus, this is the first reported case of primary bone MCL with multiple vertebral compression fractures.

MCL typically exhibits a proliferation of monotonous small- or medium-sized lymphocytes with inconspicuous nucleoli (7). The immunophenotypic analysis of MCL is usually positive for CD5, CD20, CD43 and negative for CD10 and Bcl-6 (15). The chromosomal translocation $\mathrm{t}(11 ; 14)(\mathrm{q} 13 ; \mathrm{q} 32)$ is a genetic characteristic of MCL that leads to the aberrant overexpression of cyclin D1. Cyclin D1 can be easily identified by immunohistochemistry and is considered to be the hallmark of MCL. Only in rare cases is MCL cyclin D1; in these cases, the tumor exhibits overexpression of cyclin D2 or D3 instead (16). The typical immunohistochemical features of $\mathrm{MCL}$ are $\mathrm{CD}^{+}, \mathrm{CD} 20^{+}$, cyclin $\mathrm{D1}^{+}$and $\mathrm{CD} 23^{-}$, with which the present case was in accordance.

For the present patient, PKP and biopsy were selected as the appropriate treatment methods. Kyphoplasty, which uses a dilatation balloon to restore the height of the vertebra and reduce angular kyphosis, was developed from vertebroplasty. PKP is a minimally invasive technique that induces immediate pain relief and permanent functional improvement. Despite the $\mathrm{X}$-ray and CT scan revealing that the L1 vertebra had the most severe compression fractures, indicating that it was responsible for the pain, meticulous physical examination and imageology eventually determined that T10 and T12 were the source of the pain. Thus, PKP was performed on T10 and T12. Pain relief was achieved immediately following surgery, suggesting that the procedure may comprise a good treatment option for bone MCLs with multiple, painful vertebral compression fractures.

Indolent and aggressive MCL have the worst prognosis among lymphomas, and they may have a gradually or rapidly progressive course, respectively. The indolent subgroup accounts for $10-15 \%$ of all MCL cases. Despite the fact that this MCL subgroup shows no signs of progression for a long period of time, the majority of patients face a relatively rapid disease progression, short-term response to treatment, inevitable relapses and a continuously declining survival curve, with a median survival of only 3-5 years (17-19). The following clinical factors have been associated with poor prognosis of MCL: Advanced age, poor general condition, advanced stage of the disease, splenomegaly, elevated LDH, low serum albumin, other tumors and anemia.

We propose a diagnosis of stage IV disease for the current patient, due to multiple bone involvement and the Ann Arbor classification (9). The question remains as to whether primary osseous MCL has a better prognosis than MCL with bone involvement. The patient in the present case underwent PKP and received chemotherapy. During 1 year follow-up, the patient remained in good clinical condition, only complaining of slight back pain. Although patients with one or multiple bone lesions usually respond well to combination therapy, including chemotherapy and local radiotherapy, the present study proposes that the current patient's prognosis will be poor considering the low median overall survival.

To date, as no characteristic symptoms and laboratory or radiological findings have been established for the identification of primary bone MCLs, the diagnosis of osseous MCL is challenging and may be delayed. Therefore, during differential diagnosis, clinicians should consider that multiple vertebral compression fractures without any systematic symptoms may be associated with MCL. Early diagnosis and management may contribute to the improved prognosis and survival of patients with osseous MCL.

\section{References}

1. Weisenburger DD, Kim $\mathrm{H}$ and Rappaport $\mathrm{H}$ : Mantle-zone lymphoma: A follicular variant of intermediate lymphocytic lymphoma. Cancer 49: 1429-1438, 1982.

2. Berard CW and Dorfman RF: Histopathology of malignant lymphomas. In: Clinics in Haematology. Rosenburg S (guest ed) Volume 3. W.B. Suanders, London, pp39-76, 1974.

3. Leonard JP, Williams ME, Goy A, Grant S, Pfreundschuh M, Rosen ST and Sweetenham JW: Mantle cell lymphoma: Biological insights and treatment advances. Clin Lymphoma Myeloma 9: 267-277, 2009.

4. Ferrer A, Salaverria I, Bosch F, Villamor N, Rozman M, Beà S, Giné E, López-Guillermo A, Campo E and Montserrat E: Leukemic involvement is a common feature in mantle cell lymphoma. Cancer 109: 2473-2480, 2007.

5. Tiemann M, Schrader C, Klapper W, Dreyling MH, Campo E, Norton A, Berger F, Kluin P, Ott G, Pileri S, et al: Histopathology, cell proliferation indices and clinical outcome in 304 patients with mantle cell lymphoma (MCL): A clinicopathological study from the European MCL Network. Br J Haematol 131: 29-38, 2005.

6. Kelkitli E, Atay H, Yıldiz L, Bektaş A and Turgut M: Mantle cell lymphoma mimicking rectal carcinoma. Case Rep Hematol 2014: 621017, 2014.

7. Doorduijn JK and Kluin-Nelemans HC: Management of mantle cell lymphoma in the elderly patient. Clin Interv Aging 8: 1229-1236, 2013.

8. Alwasaidi TA, Hamadah A, Altouri S, Tay J, McDiarmid S, Faught C, Allan D, Huebsch L, Bredeson C and Bence-Bruckler I: Outcomes of both abbreviated hyper-CVAD induction followed by autologous hematopoietic cell transplantation and conventional chemotherapy for mantle cell lymphoma: A 10-year single-centre experience with literature review. Cancer Med 4: 1817-1827, 2015.

9. Carbone PP, Kaplan HS, Musshoff K, Smithers DW and Tubiana M: Report of the Committee on Hodgkin's Disease Staging Classification. Cancer Res 31: 1860-1861, 1971.

10. Lai R and Medeiros LJ: Pathologic diagnosis of mantle cell lymphoma. Clin Lymphoma 1: 197-206, 2000.

11. Banks PM, Chan J, Cleary ML, Delsol G, De Wolf-Peeters C, Gatter K, Grogan TM, Harris NL, Isaacson PG and Jaffe ES: Mantle cell lymphoma. A proposal for unification of morphologic, immunologic and molecular data. Am J Surg Pathol 16: 637-640, 1992.

12. Harris NL, Jaffe ES, Stein H, Banks PM, Chan JK, Cleary ML, Delsol G, De Wolf-Peeters C, Falini B and Gatter KC: A revised European-American classification of lymphoid neoplasms: A proposal from the international lymphoma study group. Blood 84: 1361-1392, 1994.

13. Harris NL, Jaffe ES, Diebold J, Flandrin G, Muller-Hermelink HK, Vardiman J, Lister TA and Bloomfield CD: The World Health Organization Classification of hematological malignancies report of the Clinical Advisory Committee Meeting, Airlie House, Virginia, November 1997. Mod Pathol 13: 193-207, 2000.

14. Lai R and Medeiros LJ: Pathologic diagnosis of mantle cell lymphoma. Clin Lymphoma 1: 197-206; discussion 207-208, 2000.

15. Vose JM: Mantle cell lymphoma: 2013 Update on diagnosis, risk-stratification and clinical management. Am J Hematol 88: 1082-1088, 2013.

16. Rosenwald A, Wright G, Wiestner A, Chan WC, Connors JM, Campo E, Gascoyne RD, Grogan TM, Muller-Hermelink HK, Smeland EB, et al: The proliferation gene expression signature is a quantitative integrator of oncogenic events that predicts survival in mantle cell lymphoma. Cancer Cell 3: 185-197, 2003.

17. Chandran R, Gardiner SK, Simon M and Spurgeon SE: Survival trends in mantle cell lymphoma in the United States over 16 years 1992-2007. Leuk Lymphoma 53: 1488-1493, 2012.

18. Nygren L, Baumgartner Wennerholm S, Klimkowska M, Christensson B, Kimby E and Sander B: Prognostic role of SOX11 in a population-based cohort of mantle cell lymphoma. Blood 119: 4215-4223, 2012.

19. Ghielmini M and Zucca E: How I treat mantle cell lymphoma. Blood 114: 1469-1476, 2009. 